\title{
Virtual Reality As an Effective Simulation Tool for OSH Education on Robotized Workplace
}

\author{
Miroslav Janak, Tomas Cmorej, Tomas Vysocky, Marek Kocisko and Monika Teliskova \\ Technical University in Kosice, Faculty of Manufacturing Technologies with a Seat in Presov, Sturova 3108001 Presov, Slovakia
}

\begin{abstract}
In last decade, the virtual reality became a huge trend in the field of visualization not only for simple elements, but also for complex devices, their actions and processes, rooms and entire areas. This contribution focuses on the possibilities of utilization of the elements of virtual reality for educational purposes regarding the potential employees and their OSH (Occupational Safety and Health) training at the specialized workplaces. It points to the possibility of using the applications and simulations of the workplaces and the processes for better understanding of potential danger and eventual prevention options. Such an application simulates the real workplace including the technological processes and provides the user with a motion in $3 \mathrm{D}$ environment of virtual scene observing and interacting in actual work zones
\end{abstract}

\section{Introduction}

The creation of the application that includes the elements of the virtual reality it is necessary to build a bridge between the real world, which presents the basics of the data transfer on the input, and the program section for control of virtual elements. This will practically enable the combination of real and virtual space. Currently there is a lot of ways how to manage the $3 \mathrm{D}$ data using the sensors and commercial software or even some Open Source products. After considerations about several software solutions, the application that presents an output described by this paper was designed and created in two well-known software products: Blender and Unity 3D.

\section{Blender}

It is an open source software which despite its price serves as an effective tool for displaying of $3 \mathrm{D}$ computer graphics with integration of animations, rendering, motion systems and physics. As a typical Open Source product, it is created, released and supported by the community. It can be freely downloaded in full versions providing all the tools and functions. The functions integrated in Blender can be extended and upgraded by the scripts programmed in Python language. Lot of scripts is already available in the program, but many others can be implemented or programmed directly according the requirements of the application creator. Use of this software allows the integration of the formulas, equations and calculations necessary for the successful and effective run of the application. Entire programming and also operational environment can be adjusted for the needs of the application that is created. All main programming operations are handled with use of visual scripting, which is based on connecting of the programs blocks and creation of the loops that finally builds the program sections generating the logical structure of the application.

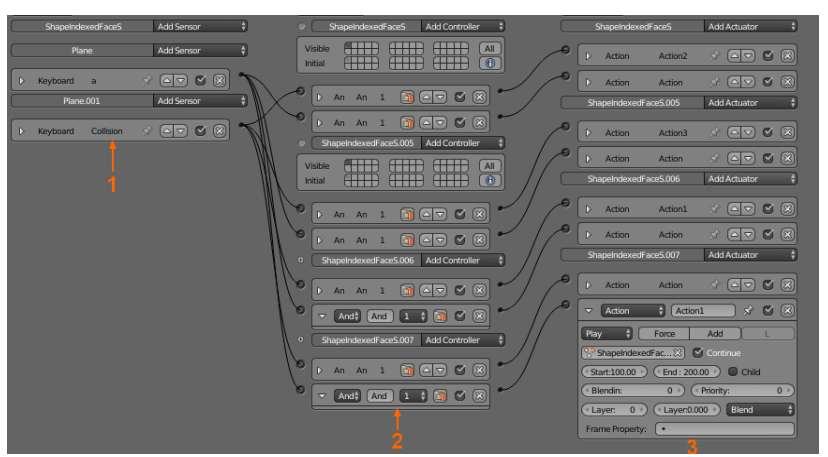

Figure 1. Programming environment of blender software

Script on Fig. 1 shows the program section that controls the settings for arms of robotic device. This script determines the actual positions and behavior (translation and rotation) of these arms which realize the continuous motion between two working units. The initiation element for the operation is selected keyboard key, which starts the complex manipulation process of the robot. In case of using the external initiation, the script can be changed Accordingly to provide the manipulation with any control device. The motions of the robot can be controlled even by the human, using the camera or Kinect device to capture and transcript live gestures.

On the figure, the first column describes the input device - initiation keyboard key. The second column builds the connection between input and output unit. 
Third one represents the motions and actions that should be realized (translation and rotation motions).

\section{Virtual environments}

For the generation of virtual environment, it is first necessary to create the 3D models of all included objects, machines, tools and fixtures that relate the manufacturing process. Such models can be modeled directly in Blender software, or (usually) imported from other graphic or CAD system. The compatibility rule must be followed when using the models from external source. Next step is to locate the machines and manipulators according to the requirements of the client and to create the connections between the input devices and the objects integrated in created environment. After creation of the compact program unit, the manipulator can realize the operations and motions assigned to it in the environment of $\mathrm{CH}$ programming language. Visual of particular devices responds to the quality and level of details for each model. The output is then presented as a whole unit which includes all the objects and their necessary motions and actions with integrated spatial dispositions and export option for further eventual use of the graphics in other software.

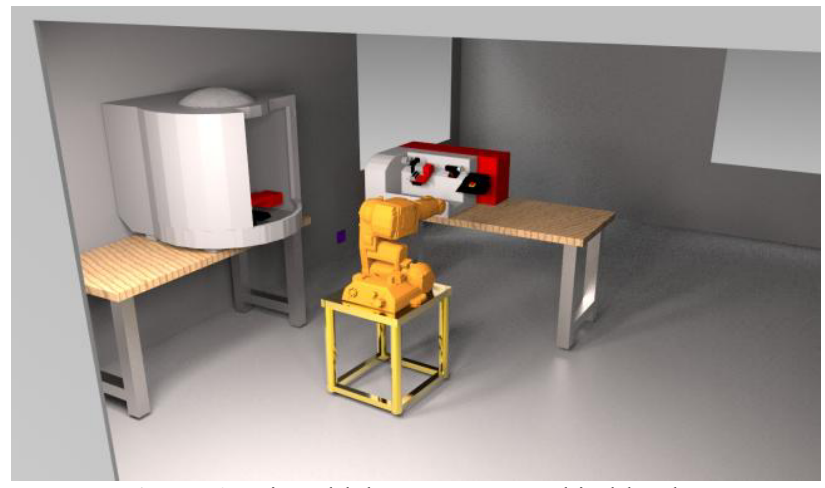

Figure 2. Virtual laboratory created in blender.

\section{Unity 3D}

This software belongs to the group of Open Source solutions for creation of virtual environment and applications. It is closely related to the Blender. It can work with programming languages $\mathrm{CH}$, Python and Javascript. It also includes the elements for modeling, so in case of need the models of new or additional objects can be created right here. It main feature though lies in scripting and creation of complex applications with all necessary parts. After importing the model from the Blender environment to the Unity all the scripts, graphics and relations are preserved and valid. So the motions and connections of particular models do not need any adjustments or settings, functions and intentions created in any previous phase of creation of the application (Blender or other software) work just fine.

The material settings and texture properties are also preserved, basically all except the lights and room attributes, which need to be created or simply selected among the existing ones. Unity provides very powerful rendering tools, which assure an excellent reproductions of colors and textures. In combination with great light calculations, this gives the user serious feeling of reality while watching the virtual animation.

Unity also handles the visual output of entire application. There are limitless options for settings of the lights, different effects and hidden interactive control elements etc.

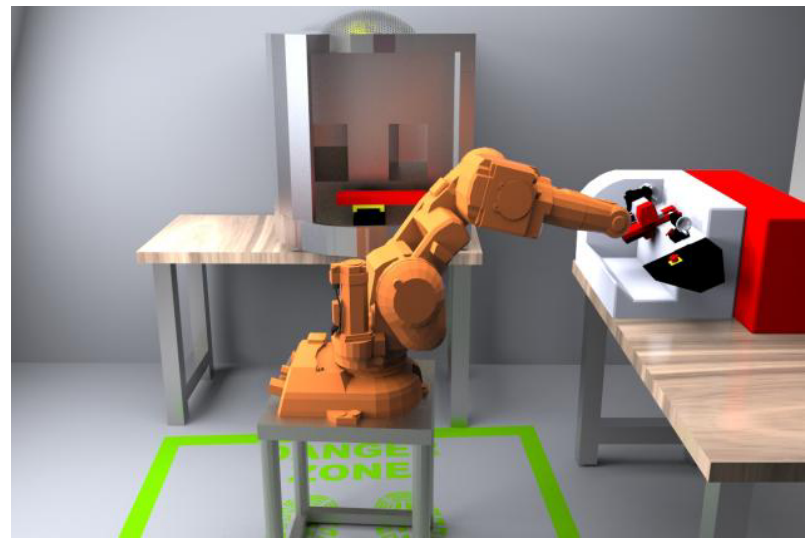

Figure 3. Working and interactive environment exported from unity software.

Output generated by the Unity software is presented as an independent application (executive file for operation on PC) or as an installation package (apk format) for devices operating on Android. While exporting for mobile devices (cellular phones) it is necessary to know the displaying parameters of the device, as it has the influence on type of glasses needed for virtual displaying. For glasses with single lens the application can run in fullscreen mode. For glasses with two lenses the application must be duplicated on the displaying surface of used device, so the user will get the proper final view.

It is hard to decide which kind of glasses for virtual reality is better. In case of the glasses with two lenses it is necessary to assure the right setting for different users regarding the eye distance and focusing. With glasses with single lens it is enough to assure possibility of the adjustment motion of the lens in the look direction, what will provide the focusing.

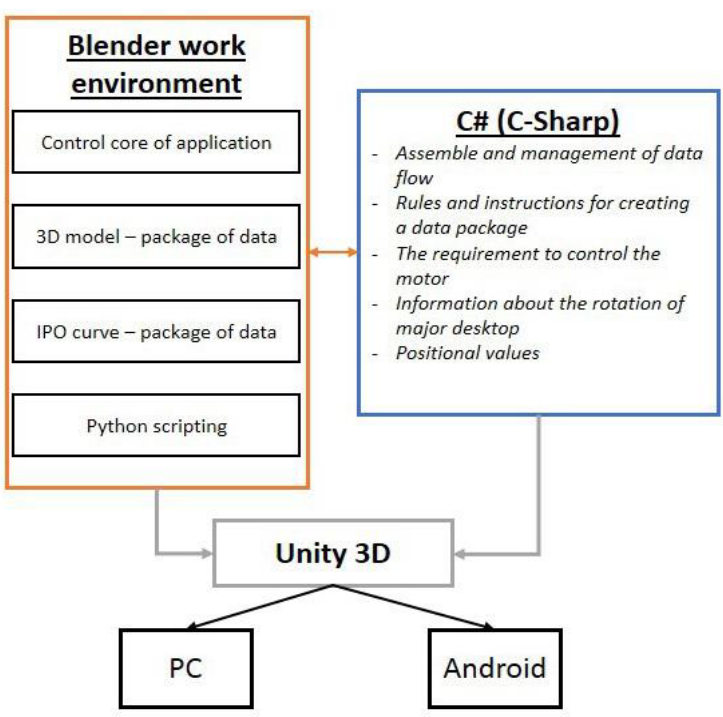

Figure 4. Logical structure of application creation process. 


\section{Occupational safety and health}

Main document for determination of applicable rules from the area of OSH in Slovakia is \# 124/2006 law Safety and Health Protection at Work. Part of this law is forcing the duty of an employer to inform their employees about the situation, facts and actions in subject field and to provide them with relevant education and training. The employer (usually through the person specialized in realization of training processes) guarantees the fulfillment of the law.

Person (trainer) selected for realization of the training and other educational activities should have relative certification obtained from National Labor Inspectorate. Experiences and praxis (or at least relative education) are often needed to be declared. The law also asks to create the sample training project of specified content and extent. This project should include the forms and methods that are planned for educational purposes. Created 3D application could be integrated into the educational process as a new form, individual demonstrational method. Individual approach would be realized using the PC, tablet or other displaying device. Each employee would have the access to the displaying device with preinstalled application and visualization of entire potential workplace. Training supervisor would determine the training modules or specific parts and workplaces from the simulation that should be taken by the employee.

\section{Application}

Proposed virtual workplace presents the real sources and equipment of the laboratories at the Faculty of Manufacturing Technologies with Seat in Presov, Slovakia. Machines are located in the working area of robotic device IRB 140 from ABB company, so the robot could manipulate with the parts and thus to simulate real working conditions. Application can be run on the computer, tablet or cellphone using either Windows or Android platform.

\subsection{Spatial motions}

Motion around the created space is provided after pressing the keyboard arrow buttons. In case of using the tablet or phone we can also employ remote controller for motion control using the Bluetooth technology. The user is situated in the environment with standard graphical and animation tools and also with features considering the physics and physical behavior of objects. Of course it laso has a definition of collision boundaries for created virtual scene. The motion around the entire scene is unlimited; limitations are presented only by the models of real manufacturing devices executing their standard tasks.

\subsection{Safety actions}

Safety actions are provided using the safety zone created in order to point out an existing danger for the user who may eventually enter the working zone with a certain risk level. If the safety zone is entered, the application gives the error message which signals that some rule has been broken. Beside this, the working cycle is stopped automatically. Safety zone is displayed as a green square shown on Fig. 5 This square located on the floor serves also as the sensor reference.

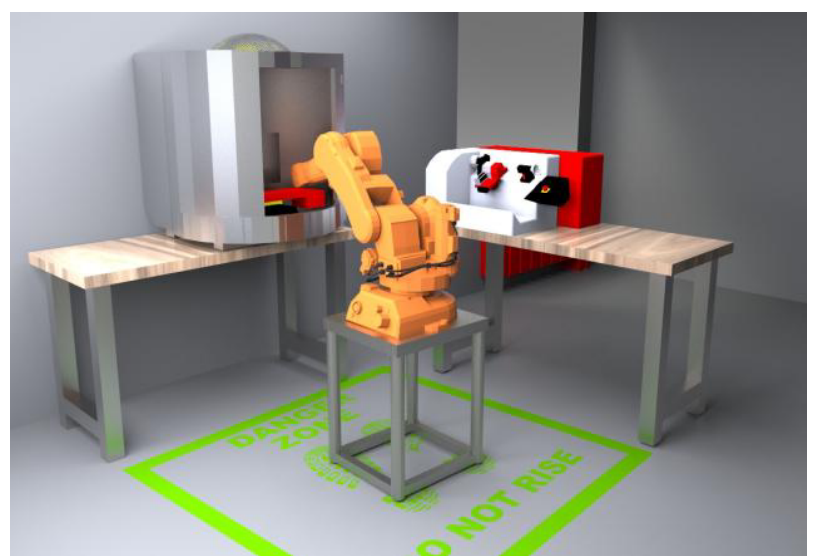

Figure 5. Safety zones for functional restraints and regulations.

The user will learn to move around the working zone without affecting the real working process by interruptions or creating any dangerous situations. Safety zones can be created in the working zones of manufacturing machines, manipulation devices or any other mechanisms, elements or systems. The zones can be also used for the definition of proper working area and for understanding of dispositional relations between all the objects, even among more employees. In case of complex mechanism and if the situation requires so, the safety zone can be created for particular section of the system which has some elements of the danger, and then to change the color of specific object after entering its safety zone. This method can be used not only for the virtual workplace but also for the simulation and visualization of entire company.

Virtual applications and applications of virtual reality provides many types of visualization features (animations, visual graphic cycles, information elements, additional audio instructing and guidance) for realization of which some constraints and logical loops needs to be created. Using them allows the user for flexible reactions to the events and situations while the application is running. After debugging, the logical core of the application realizes the generation of action of the visual element in real time without any mistakes.

Table 1 represents basic differences between the training of the employees with and without the utilization of the application for virtual reality. The users in general find this virtual way to be more attractive, as they can see the workplace they are supposed to work at yet in the educational phase in a comfortable and attractive way. Efficiency of the education is equal from the viewpoint of the output, but considering other actualities and things that can be prevented using the VR (none risk of injury for the employee and none damage caused for the company), the application exploitation is more effective. Overall time span necessary for training itself will be decreased and there is no need to get the employees to the 
operation workplace at all times, what leads to the decreasing of the potential danger in educational period. Economically, both methods are quite demanding for the employer. In case he decides for the virtual application, there are some initial investments necessary to buy the displaying devices and creation of virtual workplace. Estimated return of the costs can be expressed in the form of lower level of occupational accidents in specific time period.

Table 1. Educational methods and their strong sides.

\begin{tabular}{|l|c|c|}
\cline { 2 - 3 } \multicolumn{1}{c|}{} & \multicolumn{2}{c|}{ Training method } \\
\cline { 2 - 3 } \multicolumn{1}{c|}{} & without $\boldsymbol{V R}$ & with $\boldsymbol{V R}$ \\
\hline Atractivity & $\times$ & $\checkmark$ \\
\hline Efficiency & $\checkmark$ & $\checkmark$ \\
\hline Time & $\times$ & $\checkmark$ \\
\hline Economy & $\checkmark$ & $\checkmark$ \\
\hline
\end{tabular}

\subsection{Data collection}

During the educational process using the application the trainer can record entire activity of a trainee, including the mistakes done during specific training phase. These data can be stored in external application which could evaluate the knowledge level of the trainee regarding the safety at the workplace. On the base of data collected from more training cycles, the application should calculate the percentage of tested and verified knowledge in order to confirm the readiness of the employee for the integration in the real working process. Collected data can also be used as an input for eventual application adjustments. Therefore such recording, storing, processing and evaluation of obtained data presents high additional value to the application itself.

\section{Conclusion}

Virtual reality presents the area that finds its utilization as en effective working tool in many spheres. Currently the elements of the virtual reality are more oriented to the areas of gaming industry and advertisement, but VR can be as well used in manufacturing area. Proposed method of education of new employees removes large portion of certain actualities related to standard training process and makes the user able to learn even at home. Training personnel is able to provide, observe and supervise the training process without the need to travel directly to the subject workplace. The application possibilities of such a program are really wide, although this area needs to be better acknowledged, understood and developed.

The paper deals with the possibility to use the application of virtual reality as an effective tool for education and training of employees in the area of OSH. It describes the innovative way of teaching the employees and informing them about the potential risks and actions necessary to avoid them.

\section{Acknowledgment}

Ministry of Education, Science, Research and Sport of SR supported this work, contract VEGA No. 1/0032/12, KEGA No. 002TUKE-4/2012 and ITMS project 26220220125.

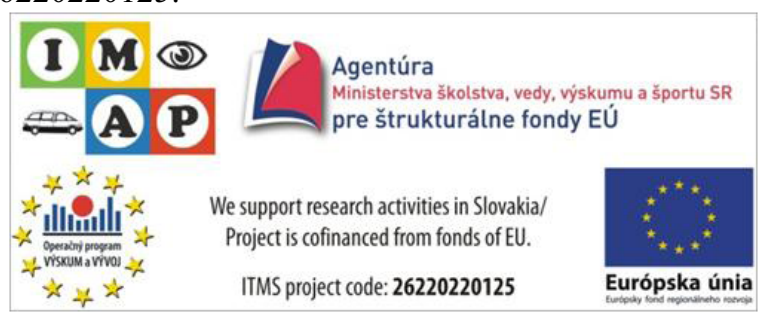

\section{References}

1. J.N. Marcinčin, J. Barna, L.N. Marcinčinová, V. Fečová, Principles of product assembly visualization by augmented reality usage, In: Annals of DAAAM for 2011 and Proceeding of the 22nd International DAAAM Symposium, Viena, 2011, 1049-1050

2. J.N. Marcinčin, J. Barna, Proceedings in Manufacturing Systems, 6, 2, 101-104 (2011)

3. J.N. Marcinčin, M. Janák, J. Barna, J. Török, L.N. Marcinčínová, V. Fečová, Verification of a program for the control of a robotic workcell with the use of AR [et al.] - 2012, International Journal of Advanced Robotic Systems, 9, 1-7 (2012)

4. J. Vallino, K.N. Kutulakos, Augmenting reality using affine object representations. Fundamentals of weareable computers and augmented reality, W. Barfield and T. Caudell (Ed.), Mahwah: Lawrence Erlbaum Assoc. Publ., 157-182

5. W.R. Sherman, A.B. Craig, Understanding of virtual reality, Interface, Application, and Design, USA, Elsevier Science, 2003 